\title{
EMPIR European project for validation of vector array SAR measurement systems
}

\author{
Djamel Allal ${ }^{*}$ \\ Laboratoire National de Métrologie et d'Essais LNE, France
}

\begin{abstract}
In the context of the new international standard IEC 62209-3 developed within IEC Technical Committee TC 106, EURAMET is running European research project Vector SAR with the aim to provide the validation methods, software tools and datasets required for traceable calibration and uncertainty analysis of vector probe array systems that automatically determine the $3 \mathrm{D}$ electromagnetic field and Specific Absorption Rate (SAR) mapping using amplitude and phase information through a 3D reconstruction algorithm.
\end{abstract}

\section{Introduction}

The specific absorption rate (SAR) is a measure of the rate at which energy is absorbed by the human body when exposed to a radiofrequency electromagnetic (EMF) field and must be evaluated during the production of smartphones.

The objective of Vector SAR project is to provide the methods, software tools and data sets necessary for the traceable calibration and uncertainty analysis of vector probe array systems (vector probe arrays that automatically determine the 3D mapping of the electromagnetic field using amplitude and phase information using a $3 \mathrm{D}$ reconstruction algorithm), which are used to measure the SAR of mobile devices.

This work is being carried out in support of the international standard IEC 62209-3 and future work on standardization of fifth generation $(5 \mathrm{G})$ devices in IEC TC 106 Technical Committee.

This project will provide a more reliable way to test compliance with IEC 62209-3 in terms of EMC exposure limits for mobile telecommunications equipment and reduce test time.

The development of mobile phones is constantly increasing. Moreover, the number of communications protocols that must be tested to assess SAR during the production of these smartphones has increased dramatically over the past decade.

As a result, the methods included in the international standards IEEE 1528, IEC 62209-1 and IEC 62209-2 now require excessively long test times to assess compliance with SAR restrictions.

For example, a modern smartphone with more than 30 integrated technologies/transmission bands would require five weeks of continuous testing to demonstrate compliance with SAR limits using the systems specified in IEEE 1528, IEC 62209-1 and IEC 62209-2 standards.
In addition, it is not reasonably possible to test all foreseeable usage patterns and the new communication standards that incorporate complex multiple-input and multiple-output (MIMO) antennas cannot be effectively evaluated using the systems specified in the current published standards, which do not address phase. Multifrequency measurement is also a challenge for traditional SAR measurement technologies, as none of them is able to distinguish between different frequencies SAR contributions.

To overcome these problems, new systems using vector probe arrays have been developed for SAR measurement. With this approach, the time required to acquire data for SAR measurement of a handset is reduced by a factor of at least 100 compared to that of a traditional single probe scanning system.

Traceable calibration methods and well quantified uncertainty estimates for these new systems must be validated.

\section{Work carried out}

The objective of providing the methods, software tools and data sets necessary for the traceable calibration and uncertainty analysis of vector probe array systems is to implement different measurement benches and data processing for uncertainty calculation.

First of all, it is necessary to set up the calibration of individual vector probes up to $6 \mathrm{GHz}$ and to check the accuracy of the measuring systems after calibration and to determine the properties of the associated phantoms (sealed phantoms).

Second, methods for the propagation of uncertainty using multivariate models should be established, applying the principles set out in the "Guide to the Expression of Uncertainty in Measurement" (GUM) [14], in particular by identifying the sources of measurement uncertainties and their propagation by

Corresponding author: djamel.allal@lne.fi 
multivariate transformations for single vector probe systems and vector probe array systems.

Finally, work is being done to verify the reliability of measurement systems and improve telecommunication signal measurement and SAR measurement for a wide range of transmitter types, as well as to develop test protocols for MIMO devices using vector probe arrays to determine the maximum SAR value (worst case) by combining MIMO signal values.

This work is being carried out to support the adoption of these new measurement systems and to contribute to the work of IEC - TC 106 Technical Committee on the successful adoption of the IEC 62209-3 standard for SAR vector measurement systems.

\section{Results}

This project develops a method and uncertainty budget for the in situ calibration of vector probes constituting a probe array, which establishes the traceability of the amplitude and phase of the electric field itself at the array measurement points. A method has been developed to transfer calibration from one antenna to another of the same type using an array system as a measuring device. A phantom prototype and a coaxial sensor have been designed to monitor the dielectric properties of the tissue simulating liquid in sealed phantoms typically used with vector probe arrays.

It has been demonstrated that the calibration process can measure the complex permittivity of the phantom with an accuracy of about $5 \%(\mathrm{k}=2)$.

The calibration of an electro-optic probe manufactured by a project partner was performed and validated using a conventional waveguide calibration system.

Reference and validation antenna models have been selected and the different antennas have been manufactured, and the corresponding data sets in terms of amplitude and phase of field distributions are being evaluated by software simulations.

This will allow reference data sets to be established from actual data in the head and body phantoms, as the currently available data are limited to SAR values of $1 \mathrm{~g}$ and $10 \mathrm{~g}$ from validation sources $(1 \mathrm{~g}$ and $10 \mathrm{~g}$ are average tissue volumes of $1 \mathrm{~cm} 3$ and $10 \mathrm{~cm} 3$, respectively, with a standardized average density of 1000 $\mathrm{kg} / \mathrm{m} 3$ for the head).

In addition, this project develops theoretical and software tools to model error propagation using complex algorithms to relate individual sensor uncertainties to the uncertainty of the average SAR of $1 \mathrm{~g}$ or $10 \mathrm{~g}$ for a given field excitation. At present, no such approach has been developed for these systems, and the GUM approach cannot be easily applied.

Work was carried out to update the uncertainty budget described in IEC 62209-3, using the S-parameter model measured by a vector network analyser for single-step and multi-step calibrations.

A model to calculate the peak SAR for a generalized measurement system based on a probe array contained in a phantom has been produced.
As this model does not fully take into account the interactions between probes, an approach is being developed to deal with measuring systems for which a detailed knowledge of the configuration used by the instrument would not be fully available, in order to ensure traceability of the uncertainty components to relevant parameters already established such as calibration of the field strength of an optical probe in a waveguide, calibration of the field strength and phase of a reference antenna using the radiated field, measured from the calibrated optical probe, and calibration of the vector probe array, by measuring the field strength of the calibrated reference antenna.

As mentioned above, the validation antennas and optical vector probes to be used for future measurements have been defined and qualified.

A set of measurements has been carried out on an electro-optic vector probe and will define the test protocol that will be used to describe the complex field distributions on a $2 \mathrm{D}$ plane resulting from a device under test, and to verify the reliability of the measurement systems.

Although reference sources have been provided to emulate real devices under test, there are currently no measurements available to compare the similarity of the field strength of a test object with the reference sources. Finally, a new effective procedure for assessing the actual exposure level, quantified by the SAR level, has been developed for MIMO devices used for beamforming [5].

This procedure provides greater flexibility to minimize the radiation and performance of these MIMO devices, compared to the traditional conservative assessment of SAR that results from the impractical testing of all antenna array states of the device with systems that do not use phase information and are less applicable to beamforming systems such as MIMO antennas.

\section{Conclusion}

The work carried out and the first results obtained will enable manufacturers of vector measurement equipment to have better control over the quality of their products and to have more confidence in the accuracy of their measurements. This will also allow the use of these systems by test laboratories to test the full compliance of mobile telecommunications devices with exposure limits. This will result in significant savings for device manufacturers and reduce the time required to bring new products to market. This will significantly reduce the costs associated with the annual calibration of the validation antenna compared to the existing methods in the standard, which offers a considerable advantage to the users of these systems.

This project will demonstrate the compliance of $5 \mathrm{G}$ devices with EMC safety limits and enhance public confidence in device safety, which is an essential step in the implementation of this new technology. This work will also facilitate the introduction of MIMO, LTE 4G, $5 \mathrm{G}$ and IoT devices by ensuring that their EMC security 
can be assessed, in line with users' increasing demands for network data capacity.

Project Vector SAR (16NRM07) has received funding from the EMPIR programme co-financed by the Participating States and from the European Union's Horizon 2020 research and innovation programme.

\section{References}

1. BIPM, IEC, IFCC, ISO, IUPAC, IUPAP, OIML, GUM, (2008)

2. BIPM, IEC, IFCC, ISO, IUPAC, IUPAP, OIML, GUM Supplement 1, (2008)

3. BIPM, IEC, IFCC, ISO, IUPAC, IUPAP, OIML, GUM Supplement 2, (2011)

4. Z. Liu, J.. Wiart, BioEM2019, S13-3, (2019)

5. L Aberbour, O Jawad, M Ramdani, P Giry, T Julien, IEEE, CAMA, 2018 